\title{
Trial puts niacin—and cholesterol dogma—in the line of fire
}

The balance of 'good' and 'bad' cholesterol noted at routine checkups-and some of the drugs used to tip this balance-might not influence heart risk in the way widely thought.

It's already known that statins, which lower levels of low-density lipoprotein (LDL), do not work for everybody. As such, doctors have long sought to complement these agents that reduce 'bad' cholesterol with medicines such as niacins and fibrates that raise levels of the 'good' stuff-namely, high-density lipoprotein (HDL) cholesterol. New evidence, however, suggests that simply elevating HDL cholesterol levels in the blood does not necessarily translate into clinical benefit for patients.

"It's a beautiful hypothesis that HDL may be cardioprotective, and there are ample preclinical as well as observation data in support of that," says Sanjay Kaul, a cardiologist at the Cedars-Sinai Medical Center in Los Angeles. "But when we put it to real test, which is the gold-standard randomized clinical trial, none of the treatments have passed muster."

The most recent failure came in May when the US National Heart, Lung and Blood Institute (NHLBI) prematurely halted the AIM-HIGH study. The 3,400-person trial, which examined high-dose extended-release niacin given together with statin therapy, was cut short after a preliminary data analysis found no additional benefits of the vitamin B-based drug in this patient population. "Maybe we've been too simplistic in thinking that raising HDL any way confers the same benefit as when it happens physiologically, and that's what we're grappling with," says the NHLBI's Patrice Desvigne-Nickens, a project officer for the trial.

"AIM-HIGH poses the most substantial challenge yet to the HDL cholesterol hypothesis," says Dan Rader, a cardiologist at the University of Pennsylvania School of Medicine in Philadelphia who was not involved in the study. Michael Davidson, director of preventive cardiology at the University of Chicago and another trial onlooker, adds, "To those of us in the field, we thought it was the right kind of study-the right patient population to test the effect of niacin-and when the trial didn't work, it was both a disappointment and a surprise."

Adding to the uncertainty, on 19 May, a week before the NHLBI pulled the plug on AIM-HIGH, an advisory committee to the US Food and Drug Administration (FDA) came to a damning conclusion on another HDL-raising drug. Off the back of the recent

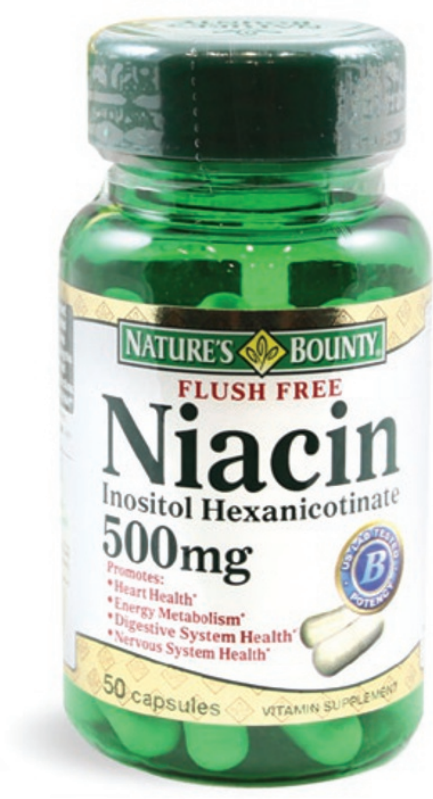

Christopher Cannon, a cardiovascular researcher at Brigham and Women's Hospital in Boston, is hopeful that newer HDL drugssuch as the cholesteryl ester transferase inhibitors dalcetrapib (from Switzerland's Roche) and anacetrapib (from New Jersey's Merck)—will provide clinical benefit. In a recently completed phase 2 safety study, his team showed that most people taking anacetrapib saw their HDL levels more than double, with only $3.3 \%$ of them experiencing heart attacks, strokes or other kinds of cardiovascular events, compared with $5.3 \%$ of those in the placebo group (N. Engl. J. Med.363, 2406-2415, 2010). Although the outcome measures are only preliminary, "it's very reassuring that it's going in the right direction," he says.

Meanwhile, many researchers are looking for better tests of HDL function to predict which drugs will succeed in the clinic. In January, Rader and his colleagues reported a measure of HDL efflux capacity - that is, the molecule's propensity to accept cholesterol from lipid-laden macrophages-that was highly associated with rates of coronary artery disease (N. Engl. J. Med. 364, 127-135, 2011). And last year, Alan Tall from New York's Columbia University published his own efflux assay that predicted a much larger benefit of anacetrapib compared to niacin (Arterioscler. Thromb. Vasc. Biol. 30, 1430-1438, 2010). Unpublished work shows a similar dramatic benefit for dalcetrapib. "This might be the best shot we have in terms of a functional biomarker that tells us whether [HDL] is working," Tall says.

And the story might not be over for niacin. Lipidologist Jeff Probstfield, an AIM-HIGH trial investigator from the University of Washington in Seattle, stresses that the target population in the AIM-HIGH study was quite specific, with particularly low levels of LDL cholesterol at enrollment, so it's hard to extrapolate the findings to the population at large. As such, researchers are eagerly awaiting the findings of another ongoing large international trial of high-dose, extendedrelease niacin called THRIVE, which is testing another patient population with more variable LDL levels, before making further clinical changes.

"That will probably be the final nail into the coffin of niacin, or it will resurrect niacin," says Prediman Shah, director of cardiology and atherosclerosis research at Cedars-Sinai. Results from the THRIVE trial are expected in 2013.

Elie Dolgin 